\title{
The Driving Science for TMT's AO Systems
}

\author{
Warren Skidmore, Lianqi Wang, and Christophe Dumas \\ Thirty Meter Telescope International Observatory, 100 W. Walnut St., Suite 300, Pasadena, \\ CA 91124, USA
}

\begin{abstract}
We highlight several potential observing programs for the Thirty Meter Telescope that impose technically challenging requirements on the performance and operation of the adaptive optics system(s). Some requirements impact the science instruments and the on-detector-guide-windows. Requirements that appear technically challenging include the time to setup the LGS/MCAO system, rapid real-time selection of guide stars for any position on the sky, use of extended $\mathrm{AO}$ guide objects, relative motions between several non-sidereal guide targets and $\mathrm{AO}$ operation in the vicinity of bright sources. Less challenging but still essential requirements include observation planning tools that allow identification of times when non-sidereal targets will pass next to suitable natural guide stars. AO performance estimates for extended guide targets and the availability of suitable AO guide stars over the whole sky are also shown.
\end{abstract}

Keywords: TMT, ELT, MCAO

\section{INTRODUCTION}

The design and development of the first light adaptive optics system for the Thirty Meter Telescope is in the final design phase, ${ }^{1}$ software development is proceeding rapidly ${ }^{2}$ and detailed planning of the observatory operations will begin soon. As such it is timely to consider the observing capabilities called for by the science programs that the future TMT user community expects to be able to carry out, and the challenges related to the AO systems to support those science programs.

In this paper we list nine observing programs that have been selected because they place challenging requirements on the operational procedures and performance of the TMT AO systems (both the first light and future systems). Four of these programs are discussed in the 2015 TMT Detailed Science Case ${ }^{3}$ (DSC), references to the appropriate DSC sections are given.

Some of the technical challenges involve the use of extended objects as AO guide targets and the rapid assessment of availability of suitable guide stars. The performance of the TMT first light AO system, NFIRAOS (Narrow Field InfraRed Adaptive Optics System) with non-point source AO guide stars has been modeled and the expected availability of $\mathrm{AO}$ guide stars has also been assessed. ${ }^{4}$

\section{TECHNICALLY CHALLENGING SCIENCE PROGRAMS}

\subsection{Dwarf galaxy radial mass profiles from proper motions}

\subsubsection{Science Goal(s) - DSC 3.1.1 \& 7.6}

By measuring the radial mass profiles for Milky Way Satellite, Classical dSph (dwarf spheroidal), Field and M31 Satellite dSph galaxies using proper motions we can:

1). Test the predictions of the $\Lambda \mathrm{CDM}$ scenario by establishing the presence or absence of cusps/cores in dwarf Spheroidal galaxies.

2). Measuring the distribution of dark matter in nearby satellite galaxies to investigate the 'Too big to Fail' problem of low numbers and low central densities of the largest satellite galaxies of the Milky Way.

Further author information: (Send correspondence to Warren Skidmore.)

E-mail: was@tmt.org, Telephone:+1 6263956956 


\subsubsection{Observations}

Measure the positions of a few hundred stars per dwarf galaxy with a near-IR imager to an accuracy of $\sim 30 \mu$ arc sec over a three-year baseline to derive the proper motions with a kinematic accuracy to $\sim 7 \mathrm{~km} / \mathrm{s}$ for stars with $\mathrm{K}<18.5$ with 60 s integration at each location.

Mosaiced or tiled observations covering up to $3000 \mathrm{Sq}$ arc mins with up to about 6000 pointings separated by $\sim 30$ arc sec, with a 60 s integration at each location.

\subsubsection{Impact on requirements for adaptive optics performance or operations}

Because of the large amount of tiling and size of move between each pointing this program requires very efficient set up of the LGS/MCAO to ensure adequate operational efficiency. An average time of $12 \mathrm{~s}$ to move and set up between each integration during a long sequence of $30 \mathrm{arc}$ sec moves and 60s integrations (this is to preserve $80 \%$ efficiency) $)^{*}$.

This program requires relative astrometric accuracy between stars in the same dwarf galaxy of 0.03 milliarcseconds.

\subsection{Probing The High-z Universe with Gamma-ray Bursts}

\subsubsection{Science Goal(s) - DSC 9.7}

Investigating the Inter Stellar Medium structure and chemical composition in the local environment of GRB's within $\mathrm{z}>6$ host galaxies by gathering absorption spectroscopy of long GRB afterglows.

\subsubsection{Observations}

ToO response time of 5 minutes from the GRB discovery (discovery is essentially instantaneous with space based facilities) is needed to execute a pre-approved set of observations at a position on the sky that is not known beforehand. Brightness changes of up to a factor 10 per minute can occur. Ideally R 1000 NIR IFU spectroscopy is needed at spatial scales close to the diffraction limit for 0.8-2.4 microns. The GRB host galaxy may not be visible, but if detected then the position of the GRB within the host galaxy will be found and provide further context on the measured ISM characteristics.

\subsubsection{Impact on requirements for adaptive optics performance or operations}

To achieve the short response time on a previously unknown position on the sky requires automated assessment of availability and selection of guide stars and efficient LGS/MCAO system setup all within the 5 minute response time. The selection of guide stars must include some backup guide stars should those initially selected turn out to be unsuitable, such as being a previously unknown close binary (see Section 2.4).

\subsection{Resolved stellar populations as tracers of galaxy evolution and reconstructing the star formation histories of nearby galaxies}

\subsubsection{Science Goal(s) - DSC 7.8.4 \& 7.8.5}

1). Perform photometry of spatially-resolved stellar populations to construct color-magnitude diagrams in galaxies out to the distance of the Virgo Cluster $(16.5 \mathrm{Mpc})$ to provide the first-ever 'archeological' sample large enough to unambiguously show the stochastic nature of galaxy assembly.

2). To investigate whether there is a metallicity dependence of the Initial Mass Function.

3). To reconstruct the star formation history for stellar systems within a range of different environments and galaxy types by analyzing their color-magnitude diagrams.

\subsubsection{Observations}

High precision near-IR J \& K photometry (at least two filters) of stars in nearby galaxies with $1 \%$ accuracy at $\mathrm{K}<28.5$ with 1 hour integrations using a diffraction limited AO fed imager with FOV 30 arc sec.

\footnotetext{
${ }^{*}$ The Observatory Requirements Document (ORD) requires 5s move and settling time for a 30arsec dither
} 


\subsubsection{Impact on requirements for adaptive optics performance or operations}

High precision photometry ( $<1 \%$ accuracy for $\mathrm{K}<28.5$ with $1 \mathrm{hr}$ integration) requires accurate point spread function (PSF) reconstruction across the field of view of the imager.

\subsection{Binary population: The binary frequency of field stars}

\subsubsection{Science Goal(s) - DSC 7.2}

1). A near-IR diffraction limited imaging survey of Milky Way star clusters to identify binary and multiple stars and those not fully resolved that have non-stellar PSFs and/or that appear as outliers or erroneous points on color-magnitude diagrams.

2). A serendipitous survey of low mass binaries in the field, carried out using the NFIRAOS Wave Front Sensor (WFS) during routine guide star acquisition and set-up where guide star catalog members that are previously unknown candidate binaries would be visually identified in the WFS output or identified due to the production of a problematic correction.

\subsubsection{Observations}

1). For the targeted survey, relatively high precision near-IR photometry of stellar clusters with $2 \%$ accuracy at $\mathrm{K}<25$ in 10 minute integrations using a diffraction limited $\mathrm{AO}$ fed imager with FOV 30 arc sec and at least two filters, preferably J \& K.

2). For the serendipitous the WFS output display and the quality of the wavefront measurement would be used to identify candidate binaries. Candidates would need followup spectroscopic and high accuracy astrometric measurements (the late could be derived from the WFS in multiple visits) over several month timescales to identify true binaries and classify the components.

\subsubsection{Impact on requirements for adaptive optics performance or operations}

1). High quality PSF reconstruction is required to identify partially resolved binary stars.

2). Automatic classification of candidate binaries seen by the WFS or at least logging of non-stellar objects in the TMT guide star catalog needs to occur (this is a modification from simply excluding non-stellar guide targets) so that followup observations can be made.

\subsection{Searching for and characterizing the putative rings around the Centaurs Chiron and Chariklo}

\subsubsection{Science Goal(s)}

The aim is to determine the structure, composition and temporal behavior of the rings around the Centaurs Chiron and Chariklo. Chiron and Chariklo have brightness of V 15.6 to 18.7 and $\mathrm{V} \sim 18.3$ (J 16.8) respectively, both have angular diameters of $\sim 0.035 \mathrm{arc} \mathrm{sec}$, whilst the rings are $\sim 0.1 \mathrm{arc}$ sec in diameter. The rings have azimuthal variations that move at the Keplerian orbital velocity around the primary.

\subsubsection{Observations}

Near-IR diffraction limited IFU observations on short timescales of $\sim 2$ mins to avoid smearing of the ring features. These are non-sidereal targets that rely on using NGS and LGS for all of the AO correction.

\subsubsection{Impact on requirements for adaptive optics performance or operations}

Because of the low brightness of the targets they are not suitable as guide stars, so observation planning tools to identify times when the target passes suitable Natural Guide Stars (NGS). To follow the target and NGS requires non-sidereal On-Instrument WaveFront Sensor (OIWFS) tracking up to a few \% of the sidereal rate.

\subsection{Measuring the chemical composition of main belt asteroids}

\subsubsection{Science Goal(s)}

To measure the chemical composition of main belt asteroids (between Mars and Jupiter). The angular sizes of main belt asteroids are $<0.007$ arc sec to about 0.6 arc sec. $\mathrm{V}$ band target brightness is $8^{\text {th }}$ to $24^{\text {th }}$ magnitude $(\mathrm{V}-\mathrm{J} \sim 2)$. 


\subsubsection{Observations}

AO fed near-IR medium resolution $(>4,000)$ spectroscopy between 1 to 2.4 microns using natural guide stars and LGS for MCAO. This can be gathered with an IFU or a long slit spectrograph though the advantage of the IFU is that in many cases the asteroid will be spatially resolved allowing the spatial variations in the composition to be mapped.

\subsubsection{Impact on requirements for adaptive optics performance or operations}

Observation planning tools that use the target's orbital ephemeris and the TMT guide star catalog to identify times when the target is passing a series of suitable NGSs. Non-sidereal OIWFS tracking up to $0.3 \mathrm{arc}$ sec/s is needed.

\subsection{Determining the properties of asteroid satellites}

\subsubsection{Science Goal(s)}

The aim is to determine the shape and the distribution of the chemical composition of asteroid satellites. The apparent radii of asteroid primaries range between 0.01 to 0.35 arc sec at closest approach to Earth ( $0.05 \mathrm{AU})$, brightness of $\mathrm{V} \sim 8$ to 20. The separation of satellites from the primaries ranges from 0.15 to 1.3 arc sec (1.5 to $\left.3.5 \mathrm{D}_{\text {Prim }}\right)$ and angular sizes of the satellites from $<0.007$ to 0.17 arc sec.

\subsubsection{Observations}

IFU observations of the satellite(s) using Sloan z' and JHK filters with a spectral resolution of R $<8000$. Orbital periods of asteroid satellites are typically between about 10 and 40 hours, the shorter cases necessitate integration times of $<2$ min to keep smearing to $\ll 1$ spatial resolution element unless the IFU field can track the satellite itself.

\subsubsection{Impact on requirements for adaptive optics performance or operations}

Non-sidereal tracking rates of up to $10 \%$ with natural guide stars, possibly using the non-point source primary asteroid $(<0.35$ arc sec) for tip-tilt in an on-detector-guide-window, will allow all viable TMT targets to be studied (faster, closer, larger targets will not need the capabilities of TMT). There will be differential motion between the primary asteroid (i.e. the potential tip/tilt guide object) and the satellite (rotation of the satellite itself is not considered here) so tracking of the instrument on the satellite science target whilst the primary asteroid $\mathrm{AO}$ tip/tilt target moves is needed.

\subsection{Mapping the surface properties of Jupiter's moon, Io.}

\subsubsection{Science Goal(s)}

The aim is to map the surface composition and temperature of Jupiter's moon, Io. Io has an angular size of $\sim 1.1$ arc sec and an brightness of $\mathrm{V} \sim 5$.

\subsubsection{Observations}

Near-IR diffraction limited IFU observations are needed and Jupiter's small moons can act as tip-tilt guide objects along with LGS when observing Io (Amalthea V 14.1, Himalia V 14.6, Elara V 16.3). Small moons have angular size of about 0.04 arc sec to 0.05 arc sec. Maximum differential non-sidereal rate of the small moons when being used as AO guide targets would be $\sim 0.012 \mathrm{arc} \mathrm{sec} / \mathrm{s}$.

\subsubsection{Impact on requirements for adaptive optics performance or operations}

1). The AO system has to have the ability to operate with large amounts of scattered light from Jupiter.

2 ). The need to differentially track AO guide objects that are moving with respect to each other and the science target, so the use of ephemerides to do realtime calculations of satellite (AKA guide target) positions is needed. $3)$. The use of non-point source $(<0.35$ arc sec) tip-tilt guide objects.

4). These would be 'time-critical observations' that are planned ahead of time for when the small moons are in the right positions. 


\subsection{Investigating the complex Jovian atmosphere}

\subsubsection{Science Goal(s)}

The goals are to measure the spatial and vertical distributions of the chemical composition $\left(\mathrm{NH}_{3}, \mathrm{CO}, \mathrm{H}_{2} \mathrm{O}\right.$, HCN, etc.), the vortices and larger scale circulation with a spatial resolution of $\sim 100 \mathrm{~km}$.

\subsubsection{Observations}

Spatially resolved ( $\sim 0.03 \mathrm{arc} \mathrm{sec}$ is $\sim 100 \mathrm{~km}$ as seen from Earth) near-infrared IFU spectroscopy (R $>3000$ over 1.1 to $2.4 \mu \mathrm{m}$ ) near the limb of the planet will allow the 3D motion of the atmosphere over to be mapped over physical scales that encompass multiple vortices. Different depths being probed with lines from different molecules.

\subsubsection{Impact on requirements for adaptive optics performance or operations}

1). The AO system has to have the ability to operate with large amounts of scattered light from Jupiter.

2 ). The need to differentially track $\mathrm{AO}$ guide objects that are moving with respect to each other and the science target, so the use of ephemerides to do realtime calculations of satellite (AKA guide target) positions is needed. Due to the effects of winds in the atmosphere, the target, i.e., a vortex or collection of vortices, will also be moving with respect to the bulk motion of the planet.

$3)$. The use of non-point source $(<0.35$ arc sec) tip-tilt guide objects.

4). These would be 'time-critical observations' that are planned for when the small moons are in the right positions.

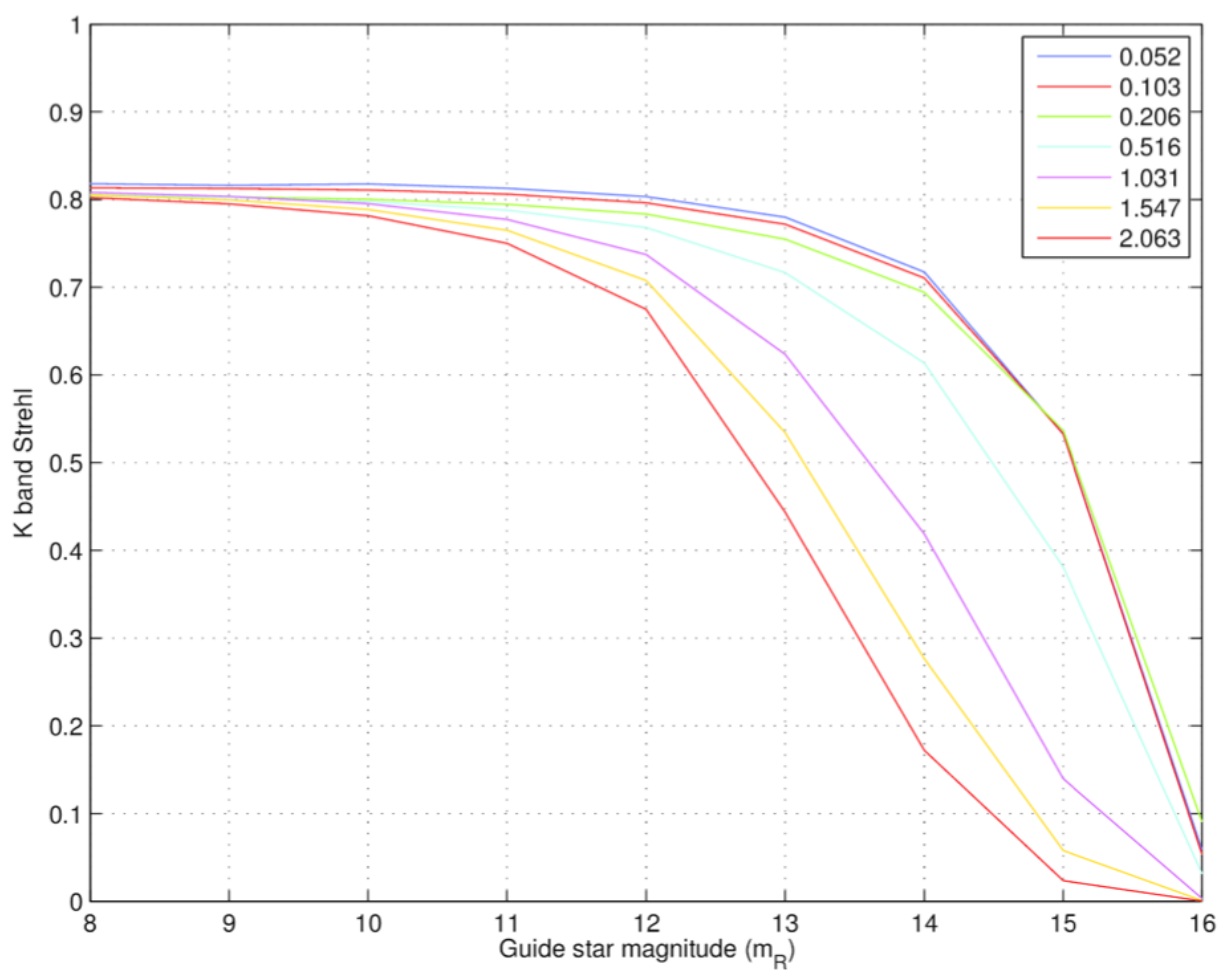

Figure 1. The modeled K band Strehl ratio when using an extended guide target in Natural Guide Star classic AO mode for different magnitude guide targets for median conditions. Colors denote different guide target diameter (arcsec) as specified in the key. 

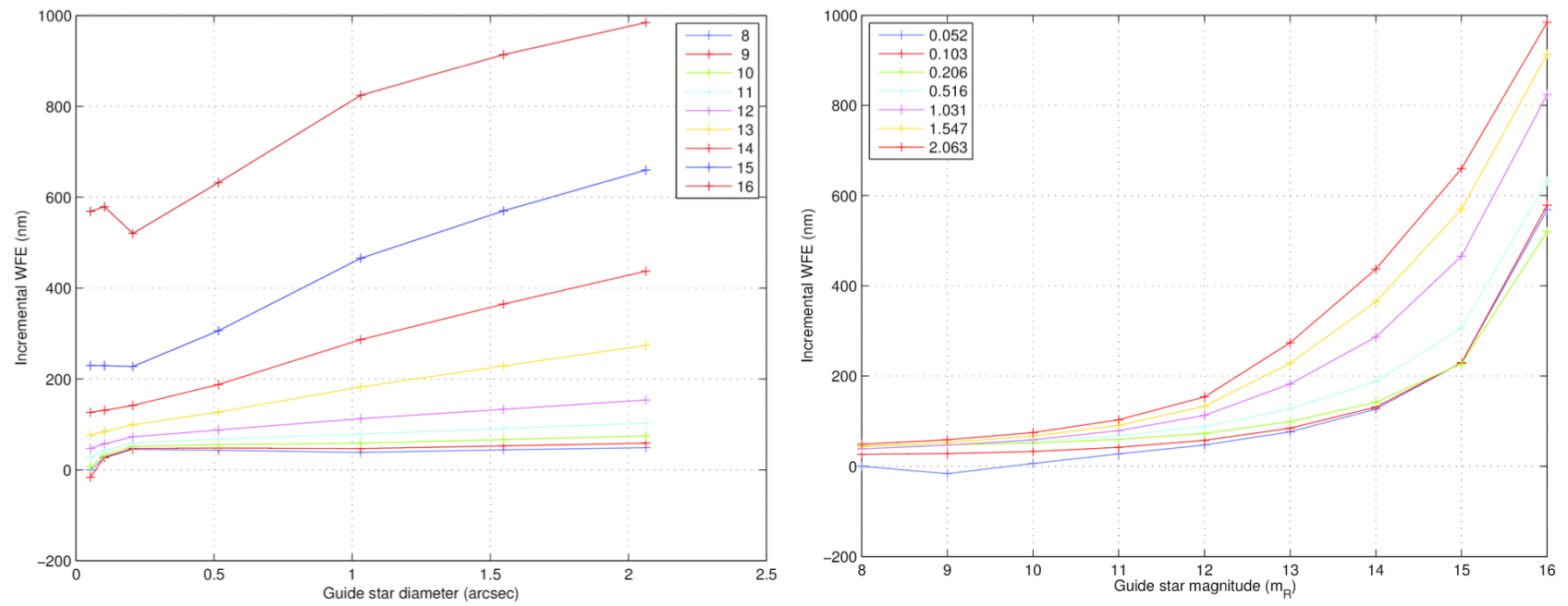

Figure 2. The modeled additional wavefront error when using an extended guide target for different magnitude guide targets. For the modeled conditions, a magnitude 8 point source gives the baseline $157 \mathrm{~nm}$ of wavefront error. The values for incremental WFE shown in this figure are in addition to that baseline. The left plot shows the model curves for different target brightness in magnitudes as a function of size, the right plot shows the model curves for different target sizes in arc sec as a function of brightness.

\section{SIMULATIONS OF AO SYSTEM PERFORMANCE AND SKY COVERAGE}

Several of the science programs described here require the use of extended objects as AO guide stars. The extent of these objects is generally 0.5 arc sec or less. To investigate the effect of using extended objects as guide targets the NFIRAOS performance was simulated. ${ }^{5}$ Figure 1 shows the expected K band strehl ratio that can be expected when using extended guide targets with a range of angular sizes and apparent magnitudes. Figure 2 shows the expected additional or incremental wavefront error encountered when using an extended tip-tilt AO guide target. For cases with guide target diameter up to 0.5 arc sec the expected performance of NFIRAOS is only slightly degraded compared to that for point source guide stars.

Some of the science programs require an excellent knowledge of the available guide stars for TMT, both to low enough enough magnitude and with high enough spatial density. The 'sky coverage', i.e. the availability of sufficient numbers of bright enough guide stars within the field of the wavefront sensors has been examined. ${ }^{4}$ We can see in Figure 3 that the sky coverage is expected to be very high, $\sim 90 \%$ averaged over zenith angles $\leq 45^{\circ}$.

\section{RELATED TALKS AND POSTERS}

Other presentations and posters at this conference that discussed the TMT AO systems include:

- Science Development for IRIS (Infrared Imaging Spectrograph) on TMT - Shelley Wright

- Thirty Meter Telescope Adaptive Optics System Error Budgets and Requirements Traceability [P1010] - Kayla Hardie

- Analyzing the Operational Behavior of NFIRAOS LGS MCAO Acquisition on the Thirty Meter Telescope using SysML [P1059] - Gelys Trancho

- Acquisition with the TMT IRIS On-Instrument Wavefront Sensor System [P2018] - David Andersen

\section{CONCLUSIONS}

We have presented several programs that impose requirements on the TMT adaptive optics systems that are technically challenging. These requirements include:

- Rapid assessment of the availability of guide stars in a previously unknown location of the sky to support ToO observations.

- Rapid set up of the LGS and MCAO system to facilitate efficient observing when mosaicing ( $>80 \%$ efficiency) 


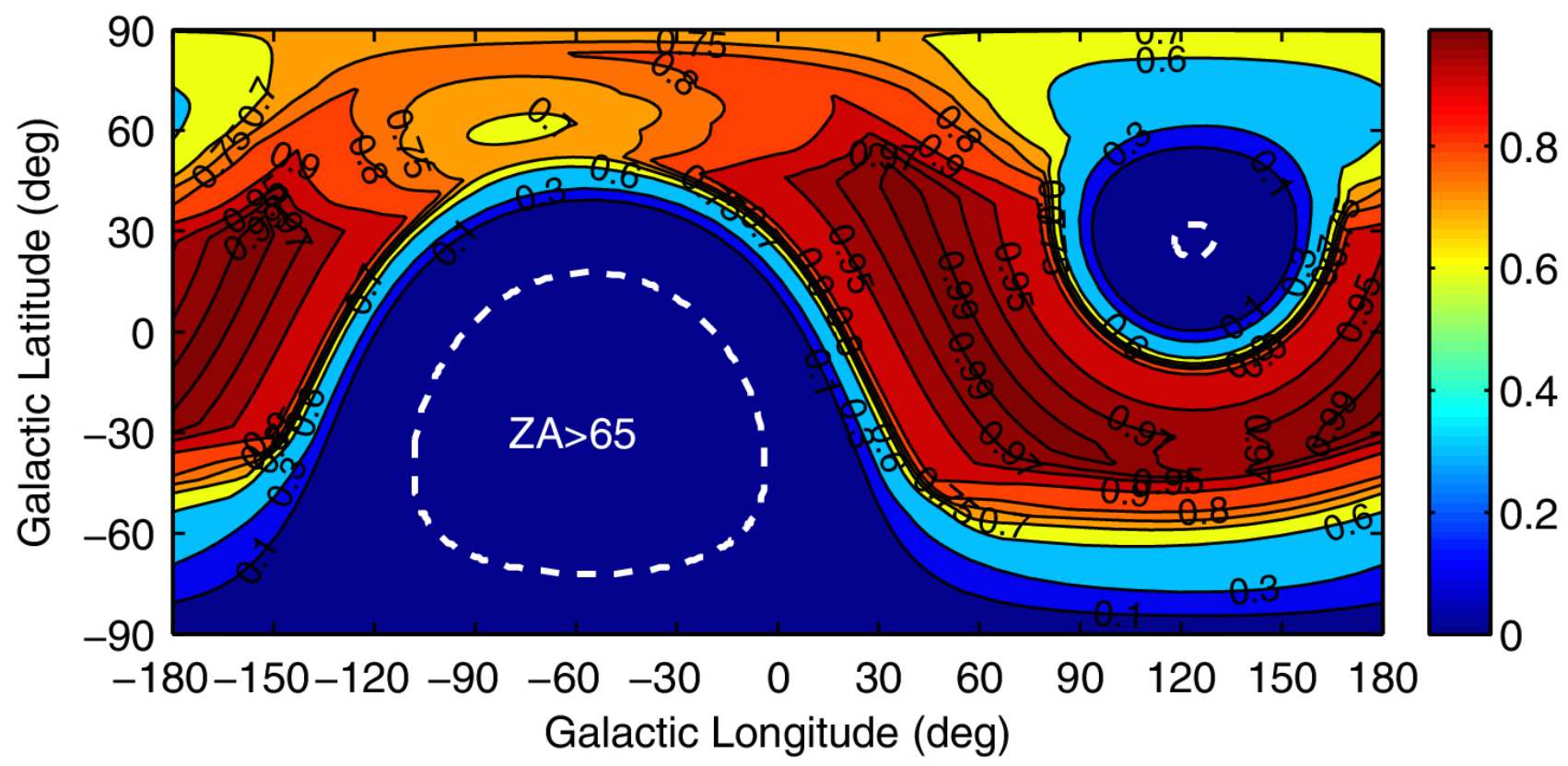

Figure 3. The probability to achieve $191 \mathrm{~nm}$ total $\mathrm{rms}$ WFE for median seeing conditions. Map of rms WFE in NGScontrolled modes in LGS MCAO mode at median sky coverage for median seeing. Hour angle is 0 h. The areas enclosed by dashed white curves are not reachable by TMT from Maunakea owing to zenith 30 angle greater than 65 . This map illustrates the probability of identifying suitable combinations of NGS for ToO programs and observations of non-sidereal targets (Figure from Lianqi et al. $2012^{4}$ ).

or rapid response for ToO observations.

- Identification and recording of non-stellar guide targets during acquisition and rapid transition to another guide star if needed when beginning a ToO program.

- Complex non-sidereal guide target and science target tracking where groups of non-sidereal guide targets move relative to each other and the science target and the science target may move relative to the bulk motion of the underlying body.

- Supporting the use of a combination of natural guide stars, laser guide stars and non-sidereal guide targets.

- The use of guide targets that are extended, typically $<0.5$ arc sec.

- Operation of the AO system in the presence of high levels of scattered light.

- Observation planning tools to identify when groups of natural guide stars are available to support observations of non-sidereal targets.

- Stringent astrometric precision requirements approaching 30 micro-arc sec on relative astrometric measurements of large numbers of stars in dense groups.

- Accurate photometric measurements (precision better than 1\%) for many stellar targets in near-IR imaging. This may be limited by PSF reconstruction.

Not specifically discussed here but seen in the ToO programs, the LGS system is expected to be available for rapid response ToO programs and a means to ensure that the LGS can be used over most of the sky for most of the time has to be included in the assessment of whether to implement a ToO program. This has already been solved by the RoboAO group. ${ }^{6}$ Also not specifically addressed but implied in some of the solar system cases is the need to use an on-detector guide window for the tip/tilt guide target and to use other slightly extended non-sidereal guide targets that may crowd the optical axis.

\section{ACKNOWLEDGMENTS}

The TMT Project gratefully acknowledges the support of the TMT collaborating institutions. They are the California Institute of Technology, the University of California, the National Astronomical Observatory of Japan, 
the National Astronomical Observatories of China and their consortium partners, the Department of Science and Technology of India and their supported institutes, and the National Research Council of Canada. This work was supported as well by the Gordon and Betty Moore Foundation, the Canada Foundation for Innovation, the Ontario Ministry of Research and Innovation, the Natural Sciences and Engineering Research Council of Canada, the British Columbia Knowledge Development Fund, the Association of Canadian Universities for Research in Astronomy (ACURA), the Association of Universities for Research in Astronomy (AURA), the U.S. National Science Foundation, the National Institutes of Natural Sciences of Japan, and the Department of Atomic Energy of India.

\section{REFERENCES}

[1] C.Boyer and B.Ellerbroek, 2016, "Adaptive Optics Program Update at TMT", Proc. Soc. Photo-Opt. Instrum. Eng., 9909, 990908

[2] H.Buur, A.Subramaniam, K.Gillies, C.Dumas and R.Bhatia, 2016, "TMT approach to observatory software development process", Proc. Soc. Photo-Opt. Instrum. Eng., 9913, 991319

[3] W.Skidmore, 2015, "Thirty Meter Telescope Detailed Science Case: 2015", Research in Astronomy and Astrophysics, 15, 1945

[4] L.Wang, D.Andersen and B.Ellerbroek, 2012, "Sky coverage modeling for the whole sky for laser guide star multiconjugate adaptive optics", Appl. Opt., 51, 3692

[5] L.Wang, J.P.Veran and B.Ellerbrock, 2017, "Pyramid WFS performance Tolerance Study for NFIRAOS", Proc. Fifth AO4ELT

[6] C.Baranec, R.Riddle and N.Law, 2017, "Automated Adaptive Optics", WSPC Handbook of Astronomical Instrumentation, submitted, arXiv, 1709.07103 